\title{
Diseño de un sistema automático de selección de frutos de café mediante técnicas de visión artificial
}

\section{Design of an Automated Coffee Selection System by Means of Computer Vision Techniques}

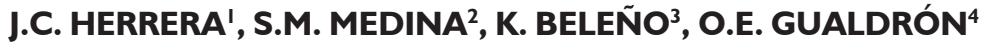 \\ ${ }^{1}$ Universidad Autónoma del Caribe, Colombia. Email: jeanhepe@hotmail.com \\ ${ }^{2}$ Universidad Autónoma del Caribe, Colombia. Email: silfri12@hotmail.com \\ ${ }^{3}$ Universidad Autónoma del Caribe, Colombia. Email: kelvin.beleno@uac.edu.co \\ ${ }^{4}$ Universidad de Pamplona, Colombia. Email: oscar.gualdron@unipamplona.edu.co \\ RECIBIDO: Febrero 26, 2015. ACEPTADO: Abril 20, 2016. VERSIÓN FINAL: Abril 27, 2016 \\ DOI: http://dx.doi.org/10.18273/revuin.v15n1-2016001 @) (1)
}

\section{RESUMEN}

En el presente artículo se propone un sistema de visión artificial para la detección del fruto de café apto para producción. Para lograr esta detección se desarrollaron dos algoritmos, uno encargado de clasificar los frutos de café en maduros o no maduros, y otro que detecta la presencia de la plaga de la broca. Para el primero se extrajeron características de color y se usó un clasificador bayesiano. Por otra parte, el algoritmo de detección de brocas busca zonas negras en la imagen, esto debido a que la evidencia dejada por esta plaga son orificios en la superficie del fruto. Además, se diseñó un sistema mecánico para el transporte de los frutos de café durante el proceso, y un mecanismo de extracción para separar los frutos, una vez estos sean clasificados por el algoritmo. Los resultados obtenidos mostraron una efectividad del $87 \%$.

Palabras Clave: Análisis de color de imágenes, Binarización, Clasificación de imágenes, Extracción de características, Fruto de café, Proceso de selección, Segmentación de imágenes, Visión artificial.

\begin{abstract}
In this Article, it is proposed a computer vision system, which can detect whether a coffee fruit is suitable for production or not. In order to achieve this detection two algorithms were developed, one to classify the coffee fruit in the ripe or unripe state; and the other to detect the presence of the 'coffee berry borer'. The first one uses a Bayesian Classifier to identify the colour of the fruit, and the second algorithm searches for the holes made by the coffee berry borer on the surface of the product. Moreover, a mechanical system was designed for the transportation and separation of the coffee fruits. In the first stage, coffees are transported as pictures of them are taken. At the end of this stage, the separation mechanism alters the path of the fruit based on the result of the classifier. The system proposed obtained an effectiveness of $87 \%$.
\end{abstract}

KEYWORDS: Artificial neural networks, Binarization, Feature Extraction, Image classification, Image color analysis, Image segmentation, Machine vision, Selection process.

\section{INTRODUCCIÓN}

En Colombia, el procesado del café se sigue realizando manualmente, a pesar del arduo trabajo que esto representa. Este proceso consta principalmente de cinco etapas: recolección, selección, lavado, despulpado y secado. El alcance de esta investigación se concentra en la etapa de selección, la cual consiste en determinar si los frutos de café son adecuados para la producción. Para esto, los caficultores tienen que analizar si los frutos del café están en un estado adecuado de maduración. Además, tienen que buscar la presencia de la broca, una plaga que afecta gravemente a estos cultivos.

Este artículo puede compartirse bajo la licencia CC BY-ND 4.0 (https://creativecommons.org/licenses/by-nd/4.0/) y debe referenciarse usando el siguiente formato: J.C. Herrera et al., "Diseño de un sistema automático de selección de frutos de café mediante técnicas de visión artificial," UIS Ingenierias, vol. 15, no. I, pp. 7-14, May. 2016. 
Algunos caficultores utilizan un método artesanal para clasificar los frutos de café, llamado 'método de flotes' o 'del tanque sifón', en lugar de revisar los granos individualmente. Este método consiste en llenar un tanque con agua y con frutos de café, de manera que los frutos de baja densidad - que no son adecuados- queden flotando y los de alta densidad -que son adecuadoscaigan al fondo del tanque, lográndose una eficacia de separación de $88,9 \%$. Sin embargo, este método exige un consumo elevado de agua $(4,7 \mathrm{~L} / \mathrm{Kg})$ que no se puede reutilizar, porque resulta contaminada durante el proceso [1]; por lo tanto, no se considera adecuado para el beneficio ecológico del café [2]. Por otra parte, si bien la clasificación manual de los frutos al momento de la recolección es efectiva para detectar frutos adecuados, esta afecta la productividad por el largo tiempo de procesado.

El objetivo de esta investigación es diseñar un sistema de visión artificial, como método alternativo a los tradicionales, capaz de seleccionar los frutos de café, basado en los criterios usados por los caficultores, el estado de maduración y la presencia de la broca. El presente artículo se encuentra organizado de la siguiente forma: (1) una sección de antecedentes con trabajos parecidos, realizados por otros autores; (2) una sección de metodología, con una descripción de los pasos realizados para llegar a los (3) resultados, en la cual se menciona la efectividad del sistema propuesto y (4) una sección de conclusiones, en donde se explican los resultados y trabajos a futuro.

\section{ANTECEDENTES}

La implementación de sistemas de visión artificial en proyectos de agricultura se ha realizado alrededor del mundo, como alternativa para la optimización de los procesos de recolección y selección de la mejor materia prima. Uno de esos proyectos es el realizado en [3], que utilizó dos métodos de clasificación: el método de comparación de plantillas y la comparación de histogramas. En este proyecto se observó que la clasificación de histogramas resulta más eficiente; sin embargo, esta arroja falsos positivos ocasionalmente. Para corregir este detalle, se sugirió estudiar los histogramas de los frutos de café en formato RGB en lugar de la escala de grises.

Investigadores del Grupo de Control y Procesamiento Digital de Señales de la Universidad Nacional de Colombia, sede Manizales, realizaron comparaciones entre tres distintas técnicas para la extracción de características de los frutos de café, con el fin de identificar los frutos en verdes y maduros. Las técnicas implementadas fueron las siguientes: filtro con base en análisis multivariado, algoritmo wrapper, y análisis de componentes principales (PCA).
Este último mostró los mejores resultados y la mejor fiabilidad. Además, se concluyó que la utilización del promedio de los píxeles en cada uno de los canales RGB es adecuado para el reconocimiento de frutos maduros [4].

Otros investigadores de la misma universidad realizaron un trabajo similar en [5]. Los autores extrajeron 208 características morfológicas del fruto del café, y de estas seleccionaron las 9 más importantes mediante el índice discriminante de Fisher, siendo estas características importantes las que caracterizan de mejor forma a los frutos de café. Para el proceso de clasificación, los autores utilizaron tres algoritmos de reconocimiento de patrones: clasificador bayesiano, redes neuronales y clustering difuso. Los resultados obtenidos por cada uno de ellos fueron los siguientes: el clasificador bayesiano obtuvo un error del $5 \%$ y la red neuronal obtuvo un $7.5 \%$. Sin embargo, el primero realizó el trabajo en un tiempo de $5.5 \mathrm{~ms}$; mucho más lento, comparado con el logrado por la red neuronal de $0.8 \mathrm{~ms}$.

Luis Silva y Sergio Lizcano diseñaron en [6] un sistema que pudiera seleccionar las frutas que presentaran el grado de maduración más adecuado para tener la fruta en su punto de mayor calidad. El sistema fue provisto con fotografías de piñas en sus diversas fases (maduras, verdes, sobre-maduradas). Lo que lo diferencia del proyecto anterior no es tanto su algoritmo de clasificación, sino el análisis de las frutas en un espacio de color diferente al convencional RGB. En su lugar, los autores utilizaron el espacio de color HSV, en el cual evidenciaron que se pueden analizar de manera más directa las cualidades que definen la maduración de la piña.

En su estudio [7], F. Guevara y J. Gómez utilizaron técnicas de visión artificial en la clasificación de los granos de trigo y cebada. La investigación consistió en un análisis de la precisión de la clasificación, de acuerdo con las características tomadas del trigo y la cebada, tales como la morfología, el color, y la textura. Los autores aplicaron el algoritmo de los $\mathrm{K}$ vecinos más cercanos, logrando un $99 \%$ de precisión en la clasificación usando los tres tipos de características mencionadas anteriormente.

\section{METODOLOGÍA}

La Figura 1 muestra el proceso general para la clasificación del café, propuesto en este artículo. En primer lugar, el café es transportado por una cinta transportadora, mientras que las cámaras del sistema de adquisición de imágenes toman capturas de la fruta, sobre las cuales actúan los algoritmos de procesamiento de imágenes. Por último, al final de la cinta transportadora, un mecanismo de selección recibe el fruto café y redirige 
su camino, dependiendo de si se trata de un grano adecuado o uno inadecuado. Cada una de estas etapas se detalla a continuación.

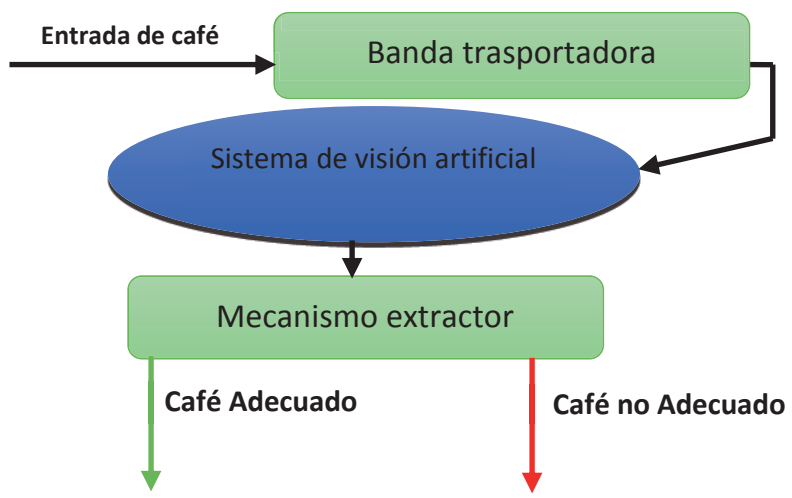

Figura I. Diagrama General. Fuente. Elaboración propia

\subsection{Sistema de Adquisición de imágenes}

Antes de diseñar el sistema de adquisición de imágenes, es imperativo conocer cómo la broca afecta al fruto de café. Esta plaga entra al fruto de café por su ombligo, como se observa en la Figura 2 [8]. Por esta razón se desarrolló el sistema de adquisición de imágenes, de manera tal que los frutos de café entren acostados, y así las cámaras, cada una ubicada en paralelo con respecto a la otra (ver flechas en la Figura 3-a), puedan analizar ambos lados del fruto.

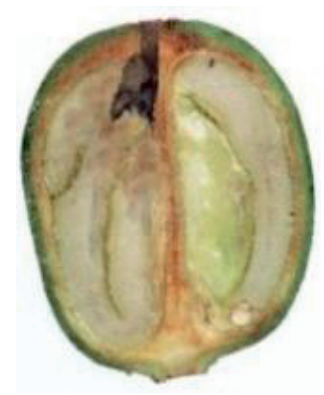

Figura 2. Broca. Fuente. Cenicafé

Como se ilustra en la Figura 3-b, para adecuar la adquisición de las imágenes se iluminaron los frutos de café con iluminación LED blanca y se utilizó un fondo blanco. Las dos cámaras empleadas funcionaban a una resolución de 640x360.

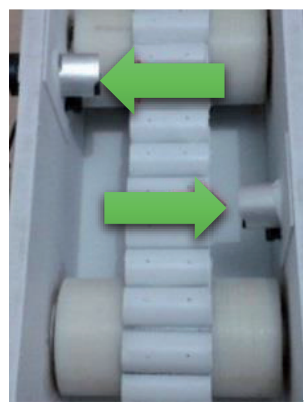

(a)

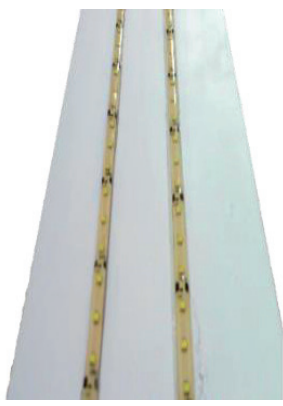

(b)
Figura 3. Sistema de adquisición de imágenes. (a) Posición de las Cámaras. (b) Iluminación. Fuente. Elaboración propia.

\subsection{Algoritmos de procesamiento de imágenes}

El algoritmo de procesamiento de imágenes inicia con la adquisición de la imagen, luego esta se analiza mediante dos algoritmos diferentes: uno de ellos busca la broca en el café y el otro clasifica a este en maduro o inmaduro. Con los resultados de los dos algoritmos, el sistema decide si el café analizado es adecuado o inadecuado para la producción. En esta investigación, los algoritmos de procesamiento de imágenes se implementaron en Python 2.7.8, usando las librerías de Open CV 2.4.9.

3.2.1. Análisis del color. El algoritmo de análisis de color consta de tres fases principales: la segmentación, la extracción de características y el algoritmo clasificador. En la primera fase, el fondo se extrae del objeto en análisis (café). En la segunda, se extraen las características. Para esto, las imágenes de espacio de color RGB se transforman a los espacios HSV y $\mathrm{L}^{*} \mathrm{a} * \mathrm{~b}$ * luego la media y la desviación estándar de las componentes $\mathrm{H}, \mathrm{S}, \mathrm{a}^{*} \mathrm{y} \mathrm{b}^{*}$ son calculadas. Finalmente el resultado de este proceso es normalizado. En la última fase, se determina si un fruto del café está maduro o sin madurar, basado en una base de conocimiento previo.

Debido a que en la etapa de conversión de los espacios de colores y extracción de características solo se necesitaba la información concerniente al café, fue imperativo pasar primero por el proceso de segmentación. Para lograr dicho proceso, se convirtió la imagen original a escala de grises, la cual era igual al promedio aritmético de las componentes RGB [9]. Luego, se utilizó el método de Otsu con el fin de encontrar el umbral óptimo para binarizar la imagen [10].

El resultado obtenido fue operado con la imagen original, con una multiplicación de elementos punto a punto. 
Como resultado se obtuvo una imagen RGB con el fruto del café y un fondo negro, de la cual se extrajo el fondo blanco.

3.2.2. Extracción de Características. Una vez segmentada la imagen se procede a extraer sus características. Para este propósito, en la investigación, se usaron los espacios de color HSV y L*a*b*, debido a que estos separan la información de color de la de iluminación, siendo más adecuados para clasificación de color [11] [12]. La información de color puede ser cuantificada con la media y la desviación estándar, pero primero se deben seleccionar las componentes adecuadas de los espacios HSV y L*a*b*. Estas componentes son: $a^{*}$, la variación entre el rojo y el verde; b*, la variación entre el amarillo y el azul; $\mathrm{H}$, el matiz de la imagen y $\mathrm{S}$, la saturación. Las componentes $\mathrm{L} * \mathrm{y} \mathrm{V}$ no se tuvieron en cuenta debido a que estas llevan la información de iluminación.

En total 8 características fueron extraídas de cada imagen; dos para cada componente de cada espacio de color. Sin embargo, los valores manejados por los componentes de los espacios de colores utilizados difieren entre sí, afectando el algoritmo de clasificación por la influencia que tienen los valores grandes sobre el clasificador [13]. Por lo tanto, a través de las ecuaciones $1,2,3$ y 4, se realizó una normalización estadística no lineal sobre las características extraídas.

$$
\begin{gathered}
\bar{x}=\frac{1}{N} \sum_{i=0}^{N} x_{i k} k=1,2, \ldots, l \\
\sigma_{k}^{2}=\frac{1}{N-1} \sum_{i=1}^{N}\left(x_{i k}-\bar{x}_{k}\right)^{2} \\
y=\frac{x_{i k}-\bar{x}_{k}}{r \sigma_{k}} \\
\hat{x}_{i k}=\frac{1}{1+\exp (-y)}
\end{gathered}
$$

La Tabla I muestra el vector de características, el cual es la entrada del clasificador. Cada imagen tiene su propio vector de características.
TABLA I

Vector De Características

\begin{tabular}{|c|c|}
\hline$\#$ & Características \\
\hline 1 & Desviación estándar componente $\mathrm{a}^{*}$ de $\mathrm{L}^{*} \mathrm{a}^{*} \mathrm{~b}$ \\
\hline 2 & Media componente $\mathrm{a} *$ de $\mathrm{L}^{*} \mathrm{a}^{*} \mathrm{~b}^{*}$ \\
\hline 3 & Desviación estándar componente $\mathrm{b}^{*}$ de $\mathrm{L} * \mathrm{a} \mathrm{a}^{*}$ \\
\hline 4 & Media componente $\mathrm{b} *$ de $\mathrm{L} * \mathrm{a}^{*} \mathrm{~b}$ \\
\hline 5 & Media componente H de HSV \\
\hline 6 & Desviación estándar componente H de HSV \\
\hline 7 & Media componente S de HSV \\
\hline 8 & Desviación estándar componente S de HSV \\
\hline
\end{tabular}

Fuente: Elaboración propia con base en [10].

3.2.3. Algoritmo de Clasificación. El clasificador utilizado en este trabajo se basa en la 'regla del mínimo error' de Bayes, que determina si un objeto pertenece a una clase si la probabilidad de pertenecer a dicha clase es mayor que la probabilidad de pertenecer a otra clase. El clasificador bayesiano es un clasificador binario, es decir, que separa el espacio de características en dos subconjuntos [13].

3.2.4. Algoritmo de detección de broca. La segmentación por umbralización se utilizó para el algoritmo de detección de broca. La imagen de entrada en escala de grises se binarizó con un umbral de 30. Los píxeles con un valor inferior a este umbral se contaron como parte de la broca y los que no cumplían con este criterio no se tuvieron en cuenta. La detección fue posible debido a la elección de un fondo blanco en la imagen. Además, los tonos claros de los frutos de café hicieron posible que las únicas zonas oscuras en la imagen fueran las brocas y las sombras proyectadas por el café.

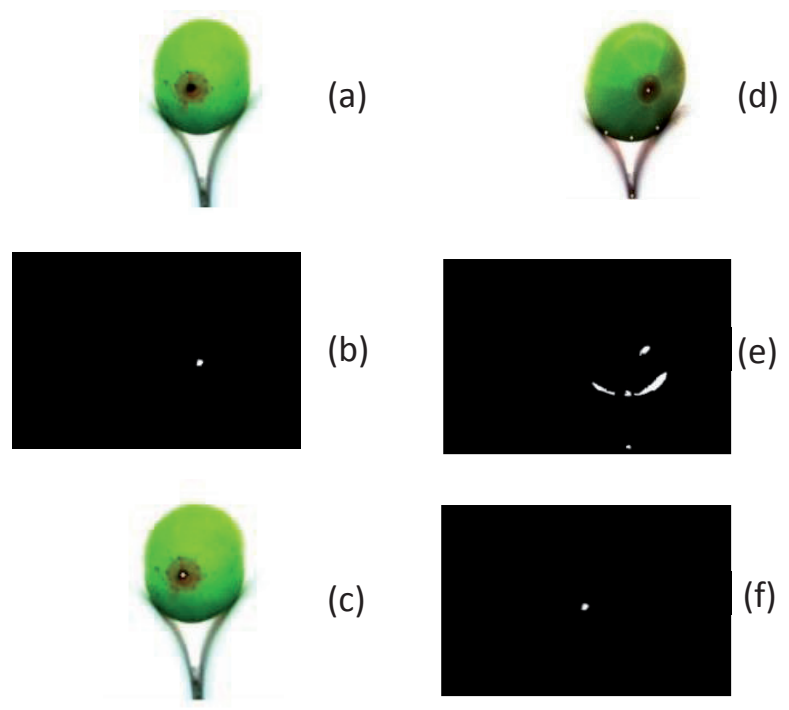

Figura 4. Detección Broca. (a) Imagen de entrada. (b) Resultado de segmentación. (c) Broca detectada. (d) Sombras detectadas como broca. (e) Resultado de segmentación de la figura d. (f) Resultado final. Fuente. Elaboración propia. 
Se seleccionó un umbral de 30, dado que las zonas negras en las imágenes tienen valores pequeños. En la Figura 4-a se observa que la imagen de entrada es un fruto de café verde con broca y en la Figura 4-b se observa el resultado de la binarización, el cual señala la broca detectada. Por último, se tiene una imagen de salida en la Figura 4-c, donde se indica con un pequeño punto la posición de la broca detectada.

A pesar de los resultados presentados anteriormente, en algunas imágenes las sombras son detectadas como brocas (ver Figura 4-d y 4-e). Para corregir este problema, se calculó el centroide del fruto del café y el de cada una de las áreas detectadas como brocas. Luego se determinó la distancia euclidiana desde el centroide del café hasta cada una de las áreas detectadas como broca, así como lo muestra la Ecuación 5.

Las áreas con una distancia de más de 40 pixeles no se consideran brocas. Por el contrario, las áreas más cerca del centroide del café se toman como tal. Las áreas blancas en la parte inferior de la Figura 4-e se han eliminado, como se ve en la Figura 4-f.

$$
d=\sqrt{\left(p_{1}-q_{1}\right)^{2}+\left(p_{2}-q_{2}\right)^{2}}
$$

3.2.5. Sistema de transporte y extracción. El dispositivo de extracción consta de dos partes: una rampa y un pequeño recipiente que se coloca sobre la rampa. El recipiente es accionado por un motor ubicado en el lado inferior de la rampa. Cuando los frutos de café caen de la cinta transportadora, se almacenan en el recipiente, en donde un sensor de color detecta la presencia del café. Una vez que el sensor marca un alto, el motor se acciona. El recipiente tiene tres posiciones definidas: una normal para recibir el café, otra a $90^{\circ}$ en el sentido de las agujas del reloj, y otra a $90^{\circ}$ en el sentido contrario, como se muestra en la Figura 5-a. Por otra parte, el dispositivo de extracción se ubica al final de la cinta transportadora como se indica en la Figura 5-b.

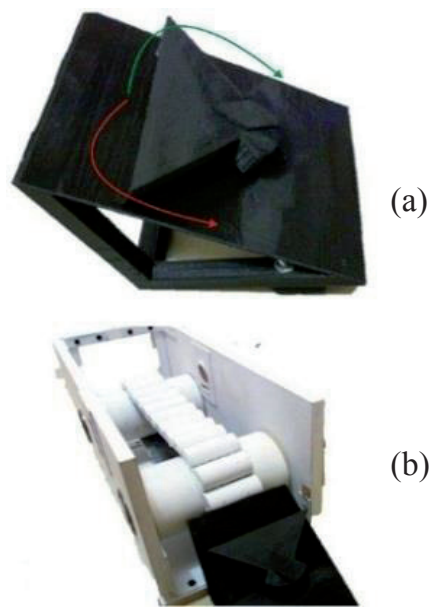

Figura 5. (a) Dispositivo de extracción. (b) Banda Transportadora y Dispositivo de extracción. Fuente. Elaboración Propia.

3.2.6. Evaluación. Para obtener el rendimiento del sistema propuesto se utilizó la curva ROC (Receiver Operating Characteristic Curve). Con ella se puede observar la relación entre las muestras clasificadas adecuadamente (PVP, Proporción de Verdaderos Positivos) y las muestras que no están clasificadas correctamente (PFP, Proporción de Falsos Positivos) [13].

El eje X de la curva ROC es representada por PFP y el eje Y por PVP. Los puntos mínimos y máximos son $(0,0)$ y $(1,1)$ respectivamente. La curva de un clasificador perfecto empieza en el punto $(0,0)$ y sube hasta la esquina superior izquierda, luego va hacia el punto $(1,1)$. Con lo anterior, se puede conocer que el clasificador que se acerca más a este comportamiento es el más adecuado.

Otra forma de medir el rendimiento del clasificador es obteniendo el área bajo la curva (AUC, Area Under Curve). En el caso del clasificador perfecto, dicha área sería igual a 1. Por otro lado, una diagonal que atraviesa la gráfica desde el origen hasta el punto $(1,1)$, indica una curva de un clasificador no funcional [14]. Tomando como ejemplo las curvas de la Figura 6, el clasificador menos adecuado está representado por la curva $B$, ya que este tiene la menor área bajo la curva y se encuentra alejada del eje Y. En contraste, la línea A representa el mejor clasificador, porque tiene mayor AUC y es la que se encuentra más cercana al eje Y. 
Some extension of Receiver operating characteristic to multi-class

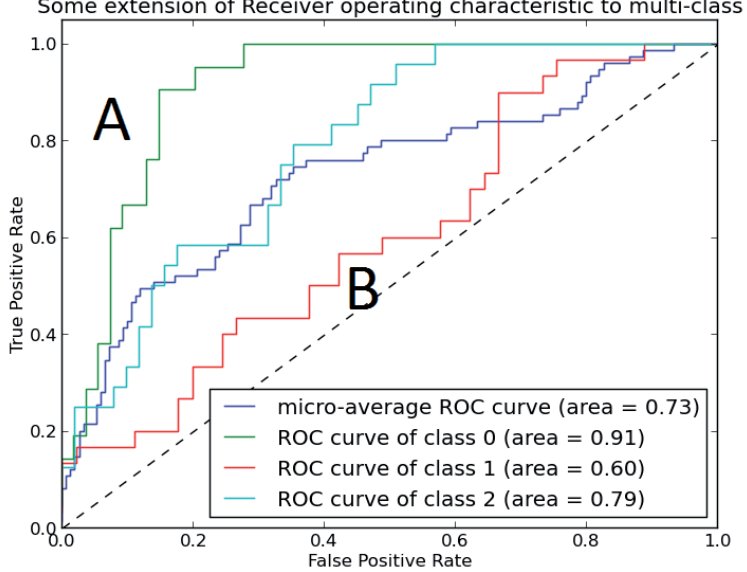

Figura 6. Curvas ROC. Fuente. Elaboración propia.

Además de la curva ROC, se utilizó una matriz de confusión, en la cual se representan las clases a clasificar. En las filas se representan los resultados reales de las clases y en las columnas los resultados del clasificador, como se observa en la Tabla 2. En este caso los verdaderos positivos son las muestras que son detectadas en mal estado correctamente; los falsos positivos son las muestras detectadas en mal estado, pero que en realidad son frutos de café adecuados; los falsos negativos, que son aquellas muestras clasificadas como adecuadas pero en la realidad están en mal estado; y por último, los verdaderos negativos, que son las muestras clasificadas correctamente como aptas para producción [15].

\section{TABLA II}

\section{Matriz De Confusión}

\begin{tabular}{|l|l|l|}
\cline { 2 - 3 } \multicolumn{1}{c|}{} & \multicolumn{1}{c|}{$\begin{array}{c}\text { Clase 1 } \\
\text { (Obtenida) }\end{array}$} & \multicolumn{1}{c|}{$\begin{array}{c}\text { Clase 2 } \\
\text { (Obtenida) }\end{array}$} \\
\hline Clase 1 (real) & $\begin{array}{l}\text { Verdaderos } \\
\text { positivos }\end{array}$ & $\begin{array}{l}\text { Falsos } \\
\text { positivos }\end{array}$ \\
\hline Clase 2 (real) & Falsos negativos & $\begin{array}{l}\text { Verdaderos } \\
\text { negativos }\end{array}$ \\
\hline
\end{tabular}

Fuente: Elaboración propia

\section{RESULTADOS}

Como se observa en la Figura 1, una vez el café entra en la banda transportadora y llega al campo de visión de las cámaras, se da inicio al procesamiento de imágenes, mediante el cual se analiza el color a través de un proceso de extracción de características y su categorización haciendo uso del clasificador bayesiano, a la vez que el algoritmo de detección de brocas determina la presencia de la plaga. Al final del proceso, se encuentra el mecanismo de separación encargado de extraer aquellos frutos considerados inadecuados para la producción.
Para comprobar el rendimiento, se tomaron en total 58 muestras de frutos de café, de las cuales 33 se encontraban en mal estado, ya sea por presencia de broca, falta de maduración o ambas; y 25 de ellas eran aptas para producción. Para conocer el estado de los frutos de café empleados como muestras se realizó una validación mediante el 'método tradicional de flotes', pues de esta manera se puede comparar los resultados obtenidos por los algoritmos con el estado real de los frutos. El sistema propuesto obtuvo un desempeño del $87 \%$.

En la curva 1, de la Figura 7, se muestra la curva ROC trazada para el clasificador de color, el cual clasificó correctamente todas las muestras, obteniendo un AUC de 1. Por otro lado, el algoritmo de detección de brocas no obtuvo tan buenos resultados, obteniendo un AUC de 0.76 (Curva 2 de la Figura 7), equivalente a un 76\% de efectividad. Cabe recordar que este algoritmo solo detecta la plaga cuando el ombligo del café se encuentra en el plano objetivo de la cámara. Por último, en conjunto, los dos algoritmos mostraron una efectividad del 87\% (AUC 0.87), tal como se puede observar en la curva 3 de la Figura 7.

TABLA III

Matriz De Confusión Del Sistema Propuesto

\begin{tabular}{|l|c|c|}
\cline { 2 - 3 } \multicolumn{1}{c|}{} & $\begin{array}{c}\text { Frutos } \\
\text { adecuados } \\
\text { (obtenido) }\end{array}$ & $\begin{array}{c}\text { Frutos } \\
\text { inadecuados } \\
\text { (obtenido) }\end{array}$ \\
\hline $\begin{array}{l}\text { Frutos adecuados } \\
\text { (real) }\end{array}$ & 20 & 5 \\
\hline $\begin{array}{l}\text { Frutos inadecuados } \\
\text { (real) }\end{array}$ & 0 & 33 \\
\hline
\end{tabular}

Fuente: Elaboración propia

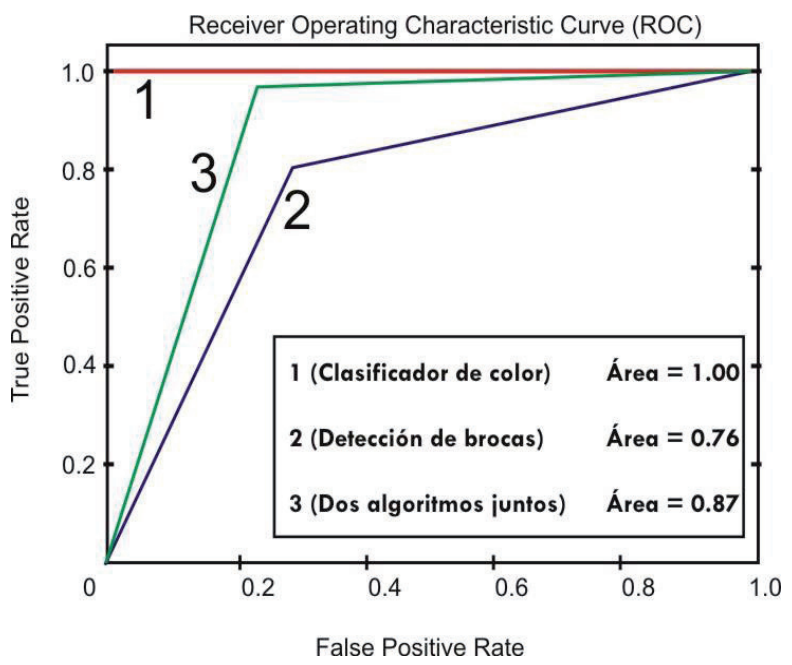

Figura 7. Curva ROC del clasificador de color. Fuente. Elaboración propia. 
Para conocer exactamente el número de muestras clasificadas como adecuadas e inadecuadas se realizó una matriz de confusión, representada en la Tabla 3. Como se aprecia claramente, el algoritmo clasificó adecuadamente todas las muestras inadecuadas; sin embargo, solo se detectaron 20 buenas, siendo en realidad 25 cafés buenos. Los 5 frutos de café restantes fueron detectados como falsos positivos, es decir, se detectaron como malos pero en realidad se encuentran en buen estado.

Partiendo del hecho de la clasificación correcta de todas las muestras, por el clasificador de color y, a partir de la curva ROC del algoritmo de detección de brocas, se puede afirmar que los errores se deben a este último. Además, al no detectarse falsos negativos pero sí falsos positivos, es posible concluir que el algoritmo está detectando brocas donde no las hay.

\section{CONCLUSIONES}

Se ha presentado un sistema de visión artificial que puede detectar frutos de café adecuados para la producción, con el cual se pretende disminuir la fatiga en los caficultores y aumentar la productividad del procesado del café. El sistema se diseñó basado en los aspectos más influyentes en la calidad de los frutos de café: el estado de maduración y la presencia de la plaga de la broca. Se desarrollaron dos algoritmos, uno basado en un clasificador de color usando la 'regla del mínimo error de Bayes', para la detección de los cafés maduros. Para detectar la broca se realizó una binarización por umbral, mediante la cual fue posible detectar los agujeros hechos por las brocas.

Ambos algoritmos mostraron resultados prometedores. El clasificador de color obtuvo una curva ROC con un AUC de 1, es decir, clasificó todas las muestras correctamente. Por otro lado, el algoritmo de detección de brocas obtuvo un AUC de 0.76. Por último, se evaluó el rendimiento de los dos algoritmos en conjunto a través de una curva ROC y una matriz de confusión, obteniendo de la primera un AUC de 0.87. Con la matriz de confusión se tuvo una vista más detallada de los resultados del algoritmo. En dicha matriz fue posible observar cuántos frutos de café fueron detectados erróneamente. El algoritmo fue capaz de detectar los 33 frutos en mal estado de la muestra, la cual estaba compuesta de 58 frutos en total. Sin embargo, se clasificaron como frutos inadecuados 5 frutos de más, que en realidad se encontraban en buen estado. Los 20 frutos restantes fueron clasificados correctamente como buenos.

Para mejorar el rendimiento del sistema propuesto se propone mejorar el algoritmo de detección de brocas y diseñar un sistema de iluminación más adecuado. Se plantean estas soluciones debido a que las fallas presentadas fueron ocasionadas por las sombras detectadas como broca. Por lo tanto, al desarrollar un mejor sistema de iluminación y un algoritmo más robusto, es posible minimizar los errores debido a las sombras.

\section{BIBLIOGRAFíA}

[1] G.I. Puerta, "Cómo garantizar la buena calidad de la bebida del café y evitar los defectos," Avances Técn. Cenicafé, no. 284, pp. 1-8, Feb, 2001. Disponible en: http://goo.gl/aWImXN

[2] C.E. Oliveros et al., "Dispositivo hidráulico de bajo impacto ambiental para limpieza y clasificación del café en cereza," Cenicafe, vol. 60, no. 3, pp. 229-238, 2009. Diponible en: http://goo.gl/0cYn7S

[3] J.A. Díaz, "Diseño de un sistema de selección de café mediante la caracterización de imágenes," ENGI Rev. Electr. Fac. Ing., vol. 1, no. 2, 2013.

[4] G. Daza, L.G. Sánchez, J.F. Suárez, "Selección de características orientada a sistemas de reconocimiento de granos maduros de café," Scientia et Technica, vol. 1, no. 35, 2007.

[5] Z. Sandoval, F. Prieto, "Caracterización de café cereza empleando técnicas de visión artificial," Rev. Fac. Nal. Agro. Medellín, vol. 60, no. 2, pp. 4105-4127, 2007.

[6] L.A. Silva, S. Lizcano, "Evaluación del estado de maduración de la piña en su variedad perolera mediante técnicas de visión artificial," Iteckne, vol. 9, no. 1, 2012.

[7] F. Guevara, J. Gómez, "A machine vision system for classification of wheat and barley grain kernels," Spanish Journ. of Agr. Res., vol. 9, no. 3, pp. 672-680, 2011.

[8] Cenicafé. Cultivemos café / Manejo Integrado del Cultivo: Manejo Integrado de la Broca [Pub. en línea]. Disponible en: http://goo.gl/IJVbSf

[9] R.C. Gonzalez, R. Woods, Digital Image Processing, 3a ed. USA: Prentice Hall, 2008.

[10] M. Nixon, A. Aguado, Feature Extraction \& Image Processing, 2a ed. Londres, UK: Elsevier Ltd., 2008.

[11] D. Savakar, "Identification and Classification of Bulk Fruits Images using Artificial Neural Networks," Int. Journ. Eng. Innov. Tech. (IJEIT), vol. 1, no. 3, 2012. 
[12] M. Hassoun, Fundamentals of Artificial Neural Networks, MA, USA: A Bradford Book, MIT Press, 1995.

[13] Z. Sandoval, F. Prieto, "Procesamiento de imágenes para la clasificación de café cereza," Prospectiva, vol. 7, no. 1, pp. 67-73, 2009.

[14] Inst. for Evidence-Based Health Prof., Edu.,The University of Georgia, "4.6: Receiver Operating Characteristic Curves" en Free Online Course in Evidence-Based Practice [Curso en línea]. Disponible: http://ebp.uga.edu/courses/

[15] Z. Sandoval, "Caracterización y clasificación de café cereza usando visión artificial," M.S. thesis, Dept. Electr., Fac. Ing. Arq., Univ.Nal., Manizales, Colombia, Abr. 2005. Disponible en: http://goo.gl /110Q38 